Frederick D. Seward

Harvard-Smithsonian Center for Astrophysics, Cambridge, Mass. and

Institute of Astronomy, Cambridge, England

\title{
ABSTRACT
}

The recent discovery of a central pulsing X-ray source makes MSH 15-52 the third SNR to contain a radio pulsar surrounded by diffuse $\mathrm{X}$-ray emission. The pulsar periods are all increasing with time and the consequent loss of rotational kinetic energy is enough, in each remant, to power a synchrotron nebula with the observed luminosity and volume.

After a review of the properties of the Crab Nebula it will be shown that both Vela X and MSH 15-52 have the same relationship between central pulsar and diffuse emission. Using empirical rules derived from these SNR, it is demonstrated that other plerionic remnants have similar characteristics. Two accretion-powered central sources can be distinguished from radio pulsars in SNR by the relatively high $\mathrm{X}$-ray luminosity of the central source compared to that of possible synchrotron diffuse emission.

\section{THE CRAB NEBULA}

The Crab contains the fastest pulsar and the brightest synchrotron nebula. Although unique in appearance it is not unique in mechanisms of energy transfer from pulsar to synchrotron nebula. Both Vela X and MSH 15-52 contain pulsars and diffuse nebulae which are probably synchrotron nebulae powered by their respective pulsars. A good summary of the Crab nebula is given by Manchester and Taylor (1977), and IAU 46 (Davies \& Smith 1971) contains much observational detail.

The distance to the Crab, $2.0 \mathrm{kpc}$ is wel1 determined for a SNR. The nebula is contained within bright optical filaments which in projection form a $5^{\prime} \times 7^{\prime}$ ellipse. The enclosed volume of $4 \times 10^{5} \mathrm{~cm}^{3}$ is filled by diffuse radio and optical emission. The X-ray nebula, however, is considerably smaller with angular dimension $\sim 1^{\prime}$ and volume $\sim 1 \times 10^{55} \mathrm{~cm}^{3}$. There is no doubt this diffuse emission is synchrotron radiation since $i \dot{t}$ is strongly polarized at all wavelengths. 405

\footnotetext{
J. Danziger and P. Gorenstein (eds.), Supernova Remnants and their X-Ray Emission, 405416.

(C) 1983 by the IAU.
} 
We consider only the X-ray data since this paper is a comparison of X-ray characteristics of different SNR. Knowing the luminosity L, and the volume, $V_{X}$; the magnetic field, $\mathrm{H}$, and energy contained in relativistic electrons, $E_{p}$, can be calculated. We assume: 1) the nebula contains a uniform field, 2) the particles all have energy such that maximum synchrotron emission is at $1 \mathrm{keV}$, the center of the Einstein HRI energy range, 3) energy in the nebula is equally divided between relativistic electrons and magnetic field.

Then: $H=25 \mathrm{~L}_{\mathrm{x}}^{2 / 7} \mathrm{~V}_{\mathrm{x}}^{-2 / 7}$ Gauss, $\mathrm{E}_{\mathrm{p}}=\mathrm{E}_{\mathrm{H}}=\frac{\mathrm{H}^{2}}{8 \pi} \mathrm{V}_{\mathrm{x}}$, and the radiation lifetime of the electrons is, $\tau=3000 \mathrm{H}^{-3 / 2} \mathrm{sec}$.

Measured and calculated properties of the Crab Nebula are 1isted in Table 2. The X-ray luminosity in the Einstein band $(0.2-4 \mathrm{keV})$ is $2 \times 10^{37} \mathrm{erg} / \mathrm{s}$ divided $95 \%$ from the nebula and $5 \%$ from the pulsar. The above formulae give: $\mathrm{H}=2 \times 10^{-4}$ Gauss, $\mathrm{E}_{\mathrm{H}}=2 \times 10^{46} \mathrm{ergs}$ and $\tau=30$ years.

Since the Crab radiates strongly at all wavelengths ranging from radio to high energy $\gamma$-rays, there is a large population of electrons ignored by the above restriction to the $\mathrm{X}$-ray band. The calculated $\mathrm{H}$ and $\mathrm{E}_{\mathrm{H}}$ are lower limits. When the entire electromagnetic spectrum is taken into account, Ltot $=2 \times 10^{38} \mathrm{erg} / \mathrm{s}, \mathrm{H} \approx 5 \times 10^{-4} \mathrm{G}$ auss and $\mathrm{E}_{\mathrm{H}} \approx 1 \times 10^{49}$ ergs. (The volume of the radio and optical nebula is $\sim 100 \mathrm{x}$ greater than that of the X-ray nebula and most of the particle energy is contained in the electrons emitting from IR - soft X-ray band. The high field is only required in the central region and $\mathrm{H}$ may well vary throughout the nebula.)

Thus if $\mathrm{X}$-rays are considered to be the only appreciable radiation the derived magnetic field is only a factor of 2.5 less than the actual field but the total energy in particles and fields is grossly underestimated.

Now consider the central pulsar which has a strong internal magnetic dipole moment, $\mathrm{m}$, and is slowing down. It radiates strong low frequency $E M$ waves and the radiation torque exerted by this magnetic dipole radiation causes the neutron star to lose angular momentum.

If the star has rotational moment of inertia I (taken as $10^{45} \mathrm{~g} \mathrm{~cm}^{2}$ ) and the period, $\mathrm{p}$, and period derivative $\dot{\mathrm{p}}$, are known; the magnetic moment is, $\mathrm{m}=\left(\frac{3 \mathrm{c}^{2}}{8 \pi} \mathrm{I} \mathrm{p \dot {p }}\right)^{\frac{1}{2}}$; the rate of rotational energy loss is, $\dot{\mathrm{E}}=-4 \pi^{2} \mathrm{I} \frac{\dot{\mathrm{p}}}{\mathrm{p}}$; and the "characteristic age" of the pulsar is, $\mathrm{A}=\frac{1}{2} \frac{\mathrm{p}}{\dot{\mathrm{p}}}$

For the Crab Pulsar $\mathrm{p}$ is the smallest of any of the $\sim 300$ known radio pulsars and $\dot{\mathrm{p}}$ is the second largest, so $\dot{\mathrm{E}}$ is high and $\mathrm{A}$ is small. Table 1 lists measured and derived characteristics of the Crab and other pulsars. 
$E \approx 5 \times 10^{38} \mathrm{erg} / \mathrm{s}$ so the Crab pulsar can supply the energy necessary to maintain the luminosity of the synchrotron nebula and the efficiency of transferring rotational energy to radiation is $\sim 0.4$. If soft $X$-rays were the only radiation observed this efficiency would appear to be only .06.

The characteristic age of the pulsar, 1230 years, is slightly larger than the known age of 920 years. The magnetic moment corresponds to a surface field of $\sim 10^{13}$ Gauss.

\section{II. $\mathrm{MSH} 15-\underline{52}$}

The X-ray and radio pulsar in MSH 15-52 is surrounded by diffuse $\mathrm{X}$-ray emission and shows promise of being truly Crab-1ike: a synchrotron nebula powered by spindown of a central pulsar. Although the diffuse emission surrounding PSR 1509-58 has not yet been proved to be synchrotron emission, the high energy X-ray spectrum (Chaippetti \& Be11-Burnel1 1982) which is hard like that of the Crab, indicates that this is likely.

The pulsar period of $150 \mathrm{~ms}$ is not unusually rapid - it is the 6 th fastest radio pulsar. The period derivative, however, is 3 times higher than that of the Crab pulsar (Table 1) and the highest of any radio pulsar. $\dot{\mathrm{E}}=2 \times 10^{37} \mathrm{erg} / \mathrm{s} ; \mathrm{m}=3 \times 10^{31} \mathrm{~cm}^{3}$ and $\mathrm{A}=1660$ years. Thus the pulsar is only slightly older than the Crab pulsar, has a magnetic moment $3 \times$ higher, and is losing energy at $\approx 1 / 30$ the rate.

The X-ray nebula observed by Seward et aZ. (1982) is much larger than that surrounding PSR $0531+21$ but not as luminous. The nebula is extended $\mathrm{N}-\mathrm{S}$ and fills a volume of $5 \times 1057 \mathrm{~cm}^{3}$. The observed $\mathrm{X}-\mathrm{ray}$ luminosity of $1.5 \times 10^{35} \mathrm{erg} / \mathrm{s}$ is only $\sim .01$ of $\mathrm{E}$ so the pulsar easily supplies the necessary energy. The calculated nebular magnetic field, $\sim 10^{-5}$ Gauss, is at least a factor of 20 below that in the Crab and characterizes the great difference in X-ray surface brightness.

The much larger envelope of MSH 15-52 allows the pulsar-supplied magnetic field to expand more freely resultting in a larger, less luminous nebula. This is probably the usual situation and a search for Crablike objects should find many like MSH 15-52 and few as bright as the Crab. This is true at least for our galaxy.

A radio map of MSH 15-52 shows only weak emission from the region surrounding the pulsar (Caswê11 et al. 1981) and probably this emission is from the expanding she11. If the synchrotron spectrum of MSH 15-52 were the same as that of the Crab except with the break in the spectrum at the X-ray band where electron lifetime equals the age of the remnant, optical brightness can be predicted to be $\mathrm{V} \approx 28 \mathrm{mag} / \mathrm{arcmin}^{2}$ and the total radio signal expected to be only $10^{-3} \mathrm{Jy}$. Thus the synchrotron nebula of MSH 15-52 could easily be much too faint to be seen at radio and optical wavelengths. 
III. VELA X

The SNR Vela $X$, containing bright optical filaments and several regions bright in soft (presumably thermal) X-rays, is only $\sim 400 \mathrm{pc}$ distant. The characteristics of PSR 0833-45 and the diameter of the shell both indicate an age of $\sim 1 \times 10^{4}$ years. The pulsar is surrounded by X-ray nebular emission (Harnden 1983) which we will treat as showing 2 distinct phenomena.

First there is the Einstein HRI observation which shows a sma11, $1^{\prime}$ diameter $(0.1 \mathrm{pc})$, nebula surrounding a point-like source coincident with the position of PSR 0833-45. The unresolved source is probably the pulsar itself although the X-rays are not pulsed. The X-ray luminosity of the small surrounding nebula (herein called Vela A) is only $5 \times 10^{32} \mathrm{erg} / \mathrm{s} .3 \times 10^{-5}$ that of PSR $0531+21$ and $2 \times 10^{-2}$ that of PSR 1509-58. If either of these more luminous pulsars were surrounded by an identical nebula, the nebular contribution would be too small to be observed. Vela A then, might indicate a process present, but not observed, in the vicinity of both the Crab and MSH 15-52 pulsars.

On the other hand, if emission with extent $0.1 \mathrm{pc}$ and strength equal to that of the emission from the PSR itself, were present, it would be easily seen as an apparent steady flux from the PSR even if spatially not resolved at a distance of 2 or $4 \mathrm{kpc}$. Since both the stronger pulsars appear $100 \%$ pulsed in the HRI, strong phenomena analagous to Vela A have not yet been distinguished in the Crab Nebula or in MSH 15-52.

The second phenomenon in Vela $X$ is nebular emission $\sim 1^{\circ}(7 \mathrm{pc})$ in extent and surrounding the PSR. We suspect that much of this emission is powered by the pulsar, based solely on the evidence of spatial arrangement, although an accidental superposition of pulsar and a region of bright thermal emission cannot at present be excluded. Spectra from the Einstein detectors should provide an answer. We note that Smith (1978) found a high energy nebula of extent $2^{\circ}$ around the pulsar and that the $2-10 \mathrm{keV}$ flux from this region measured by Pravdo et al. (1978) is higher than that expected from the pulsar alone. For present purposes we will assume that alZ X-rays from this larger nebula, Vela $B$, are synchrotron in origin and as before derive the magnetic field. Results are listed in table 2. The field is only $4 \times 10^{-6}$ Gauss, close to the average interstellar field of $3 \times 10^{-6}$ Gauss. The $\mathrm{X}$-ray luminosity, $\approx 10^{34} \mathrm{erg} / \mathrm{s}$, is easily maintained by the pulsar rotational energy loss, $\approx 7 \times 10^{36} \mathrm{erg} / \mathrm{s}$, and we conclude that Vela $B$ is analagous to the Crab synchrotron nebula.

As for MSH $15-52$ the radio and optical brightness of Vela $A$ and Vela $B$ can be predicted. Predicted radio and optical emission from Vela B are too weak for detection. Vela A, however, is perhaps detectable optically with predicted signal $\mathrm{V}=15 \mathrm{mag} / \mathrm{arcmin}^{2}$. 
Diffuse $\mathrm{X}$-ray emission has been found around solitary radio pulsars and results are summarized by Helfand (1983). The extent and luminosity $\left(10^{30}-10^{33} \mathrm{erg} / \mathrm{s}\right)$ are close to the properties of Vela A. These small nebulae are closely coupled with the pulsar itself. The relatively strong magnetic fields, small size, and short particle lifetimes make it unlikely that emission from these nebulae is influenced by the existence or non-existence of a SNR shell.

\section{THE CENTRAL PULSARS}

The X-ray emission from the three central pulsars themselves is a topic of great interest and is discussed by Bignani \& Hermsen (1982), Seward \& Harnden. (1982) and by Manchester et al. (1982). PSR 0531+21 radiates essentially the same double pulse at all frequencies from radio to $\gamma$-ray. PSR 0833-45 shows a double pulse at $\gamma$-ray energies, a single radio pulse, and in the $X$-ray band the emission is apparently not pulsed. $\mathrm{X}$-rays from PSR $1509-58$ are $\sim 100 \%$ pulsed with one pulse/cycle and there is also one radio-pulse cycle. The variety of pulse shapes from these three pulsars is difficult to interpret although the similarity of the $\gamma$-ray pulses from $0833-45$ to those of $0531+21$ is significant since 0833-45 radiates most strongly at $\gamma$-ray energies.

Pulsar properties are listed in table 1 and Fig. 1 shows both pulsar and diffuse luminosity as a function of $\dot{E}$. Both soft $X-r a y$

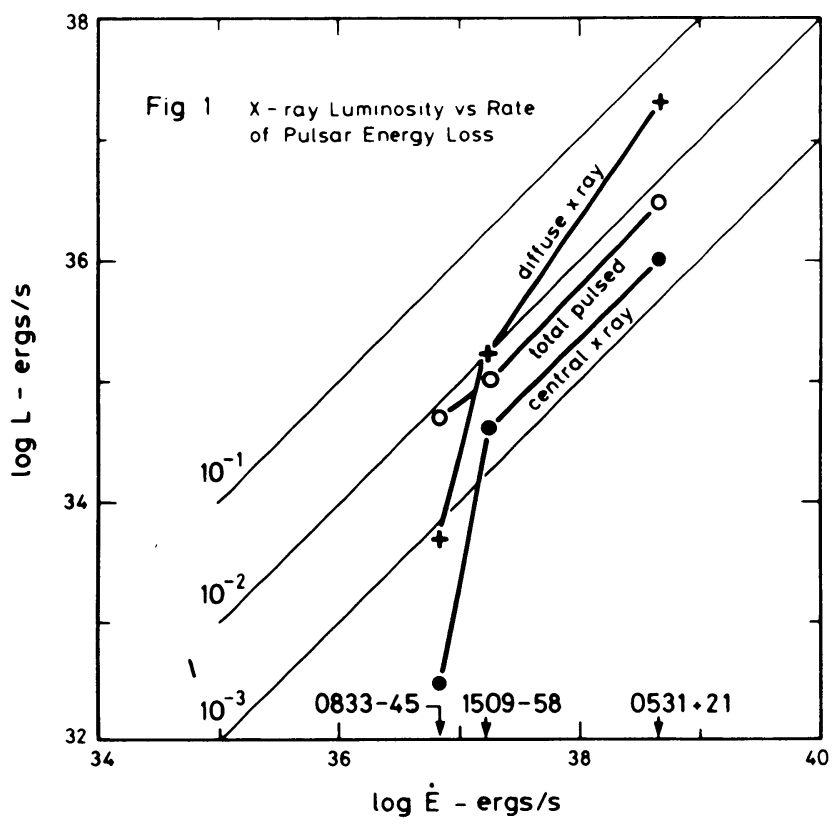


and total (radio to $\gamma$-ray) luminosity are given when known. Considering only $\mathrm{X}$-rays we note that $\mathrm{L}_{\mathbf{X}}$ (pulsar) is always less than $\mathrm{L}_{\mathbf{X}}$ (diffuse) by a factor of $4-20$, and that the efficiency for generating diffuse synchrotron $X$-rays decreases rapidly from $\sim 5 \%$ in the Crab nebula to $\sim 10^{-3}$ in Vela $X$. Including radio and $\gamma$-ray luminosity, the pulsed emission in all three cases approaches $1 \%$ of $\dot{\mathrm{E}}$ and the diffuse synchrotron luminosity of the Crab rises to $\sim 50 \%$ of $\dot{\mathrm{E}}$.

The dependence of pulsed luminosity on $\dot{\mathrm{E}}$ points to the magnetosphere of the pulsar as the origin of the radiation. The other possible mechanism, radiation from high emissivity regions on the surface of the neutron star, is less likely to be connected with $\dot{\mathrm{E}}$.

A pulsar appearing as a point-like source of unpulsed X-rays either has a high surface temperature, is surrounded by a small unresolved diffuse nebula, or is oriented so emission which would appear pulsed. from another direction appears unchanging with time. The weak pointlike X-ray emission from PSR 0833-45 could be any of these. Again, as for Vela A, if unpulsed emission this weak were present in $0531+21$ or 1509-58 Einstein would not have been capable of detecting it.

\section{FILLED-SHELL (PLERIONIC) SNR AND OTHER SNR WITH CENTRAL OBJECTS}

In the radio band a number of SNR have characteristics similar to the Crab Nebula: There is no she11-like structure, emission is polarized and maximum at the cente $r$, and the radio spectrum is $\sim$ flat. These have been 1 isted and their radio properties studied by Lockhart et al. (1977) Weiler \& Shaver (1978), and Caswel1 (1979). Four of these were observed by Einstein and X-ray results are given by Wilson (1980), Becker \& Szymkowiak(1981), and Becker, Helfand, \& Szymkowiak(1982). Diffuse $\mathrm{X}$-rays were seen from all four and, like the Crab Nebula, X-ray emission was strongest at the center, the diffuse $X$-ray source was smaller than the radio $\mathrm{SNR}$, and in the one case where a good spectrum was obtained (3C58) there was no evidence for line emission. The X-ray morphology of the central regions of $3 \mathrm{C} 58$ and of CTB80 is sharply peaked indicating the existence of a central source, and accurate locations for these sources were obtained. No point-like $X$-ray sources could be separated from the diffuse emission observed from G21.5-0.9 or from G74.9+1.2 and upper limits are given. Pulsations were not observed from any of these central regions because the flux from all the sources is below the threshold for detection of pulsations. The HRI counting rate of central objects in SNR is listed in Tables 1 and 2 . The 4 plerionic radio remnants are all below $.003 \mathrm{c} / \mathrm{s}$, a factor of $>3$ below PSR 1509-58, the weakest pulsar detected. There were just not enough X-rays collected to make a reasonable search for regular pulsations. 15,000 sec at $.002 \mathrm{c} / \mathrm{s}$ yields only 30 events. These SNR are prime targets for a more sensitive high resolution instrument which should be able to resolve central objects from surrounding diffuse emission and to find the period if emission is pulsed as in PSR 1509-58. 
Table 2 lists the observed and calculated properties of these SNR. As before, we have used the luminosity and volume of the X-ray sources to derive a magnetic field strength.

The remnants: W28, Kes 27 , and $W 44$ have also been observed by Einstein. They appear as filled-shell X-ray sources with no definite indication of a central source and are not included in this review because the data are not published or because the radio remnant is not filled-shel1 (Lamb \& Markert 1981).

The remnant RCW 103 has a central point-like X-ray source and no obvious surrounding synchrotron nebula. The central source is too weak to reasonably search existing data for regular pulsations, and the apparent absence of a synchrotron nebula would favor radiation from the surface of a hot neutron star or a background object not connected with the remnant. Since the optical candidates for the central source are all very faint, Tuohy \& Garmire (1980) favor the neutron star explanation.

Emission from the $8^{\prime}$ diameter she11, however, produces 300 times more counts in the Einstein HRI than does the point-like source, and this strong emission from the shell could easily obscure a faint synchrotron nebula. Fig. 1 and the measured $\mathrm{L}_{\mathrm{X}}=1.5 \times 10_{37}^{34} \mathrm{erg} / \mathrm{s}$ of the point source can be used to preduct a pulsar $\approx 1.5 \times 10^{37} \mathrm{erg} / \mathrm{s}$ and a diffuse nebula with $\mathrm{L}_{\mathrm{X}} \approx 1 \mathrm{x} 10^{35} \mathrm{erg} / \mathrm{s}$, only $1 / 50$ the luminosity of the shell. Thus radiation from the magnetosphere of a pulsar with small $\dot{E}$ is stili a definite possibility for the X-ray emission mechanism of this central source.

\section{A SIMPLE MODEL}

How do the magnetic fields calculated for the various synchrotron nebulae compare with expectations? Consider the following simple model. A spherical SNR of radius $R$, contains a central pulsar with rate of rotational energy loss $\dot{E}$. This energy input is equally divided between magnetic field and relativistic charged particles. The dynamics of the shell are completely determined by the initial explosion and the ISM rather than by internal energy deposited by the pulsar. Assume that the field is not particularly ordered so magnetic pressure is $\frac{1}{3} \frac{\mathrm{B}^{2}}{8 \pi}$ and that expansion of the remnant is adiabatic. Conservation of energy leads to the expression $3 \dot{\mathrm{E}}=2 \mathrm{BR}^{3} \dot{\mathrm{B}}+4 \mathrm{~B}^{2} \mathrm{R}^{2} \dot{\mathrm{R}}$.

Now assume $\dot{E}$ is constant, and that radiation energy losses are negligible. Neither is correct but the two effects do go in opposite directions and, at this point, we need to understand the gross properties.

The solution, written to explicitly contain the age of the remnant, $t$, is $B=\left(\frac{f}{R^{3}}\right)^{\frac{1}{2}}$ where $f$ is a constant dependent on the variation of

$\dot{\mathrm{R}}$ with time. 
If the shell is stationary, $\dot{R}=0, f=3$, and internal energy $=\dot{E} t=\frac{B^{2} R^{3}}{3}=2\left(\frac{B^{2}}{8 \pi}\right)\left(\frac{4 \pi}{3} R^{3}\right)$ which is exactly correct since energy is distributed equally in field and particles. If the shell motion is a free expansion, $f=3 / 2$, and the field is smaller since some energy is lost doing work. If the remnant is in the Sedov phase, $\dot{R}=\frac{2}{5} \frac{R}{6}$, and $f=\frac{15}{7}$ Since the expansion slows with time not as much energy goes into work.

These examples are plotted as straight lines in fig. 2 and compared with equipartition fields derived from observations. The points are of varying quality. The Crab, MSH 15-52, and Vela SNR points are good since $\mathrm{E}$ and $t$ are known or calculated from the pulsar properties. The uncertainty shown for MSH $15-52$ is due to the irregular nature of the. she11, which in the $\mathrm{E}$ and $\mathrm{S}$ is $18 \mathrm{pc}$ distant from the pulsar but in the NW the dense region RCW 89 is only $\sim 8$ pc distant.

The other points are considerably more uncertain: We have assumed that 3 C58 is the remnant of SN 1181 and have set the age at 800 years. $\dot{\mathrm{E}}$ was estimated from the X-ray luminosity of the central object under the assumption that Fig. 1, based on the 3 Crab-like remnants, shows a general relationship between $\dot{\mathrm{E}}$ and $\mathrm{L}_{\mathrm{X}}$. The ages of $\mathrm{G} 21.5-0.9$ and of G74.9+1.2 are estimated from the size of the remnant and as

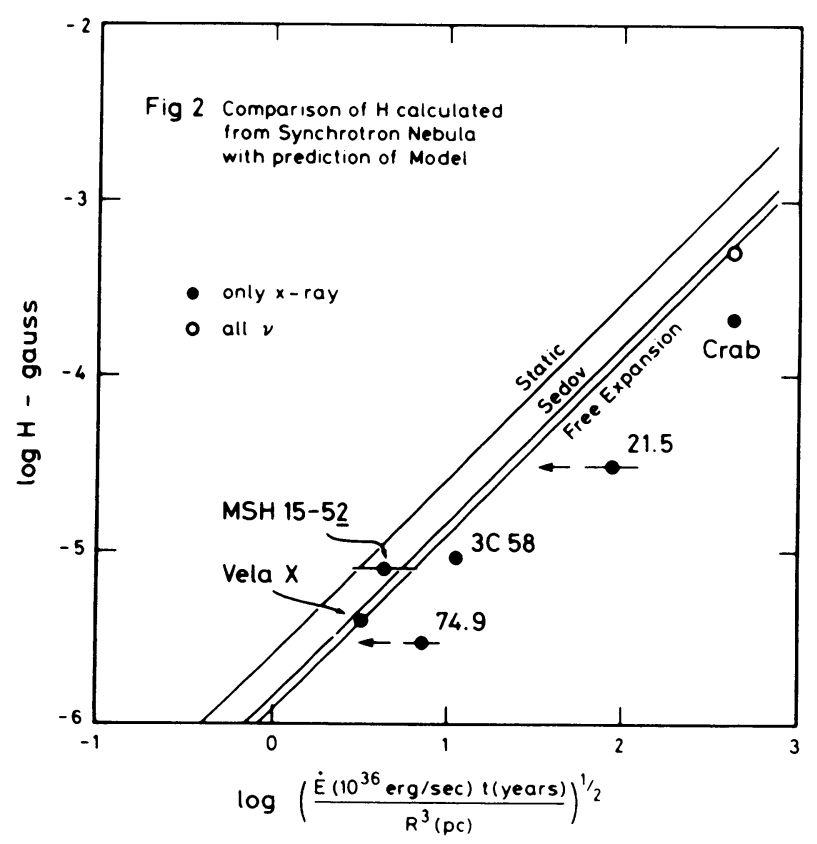


$0.5-1.5 \times 10^{3}$ years and as $0.5-1.5 \times 10^{4}$ years respectively. Since no central sources were detected $\dot{E}$ estimated for these 2 remnants are upper limits. CTB 80 is not plotted. The shell of this object is so irregular that it is difficult to determine a size, or even to identify it as an SNR with confidence.

Here is a way of organizing these data. Note that: the X-ray derived fields are not far from and generally below the model prediction. The properties of these X-ray synchrotron nebulae are determined both by the energy input from the pulsar and by the expansion of the SNR shell. The high field in the Crab is a consequence of both the high energy input of the pulsar and of the low expansion velocity of the filaments which confine the radiated pulsar energy to a small volume, resulting in a uniquely bright synchrotron nebula.

\section{TWO ACCRETION-POWERED CENTRAL SOURCES}

Both W50 and CTB109 contain central compact X-ray sources which are members of binary systems. The $3.5 \mathrm{sec} X-r a y$ pulsar within CTB109 shows frequency changes indicating orbital motion with a period of 1.9 hours (Gregory 1983). SS433 in the center of W50, is a bright optical source showing a period of $\approx 13$ days (Crampton et al. 1980).

W50 contains 2 lobes of diffuse X-ray emission which because of their orientation along the long axis of the radio nebula are almost certainly connected with the high velocity beams of SS433 (Watson et al. 1983). The emission mechanism has not been determined but, assuming synchrotron radiation, the magnetic field and energy in particles can be determined as before. Results are in table 2. X-ray luminosity of each lobe is $5 \times 10^{34} \mathrm{erg} / \mathrm{s}, \mathrm{H}=3 \times 10^{-6} \mathrm{G}$, and energy in field + particles in each lobe is $5 \times 1046$ ergs.

CTB109, a SNR with X-ray bright shell on the eastern side only and with an X-ray bright central source, contains a diffuse feature interior to the shell and pointing at the pulsar $L_{x}=8 \times 1024 \mathrm{erg} / \mathrm{s}$. Assuming this "jet-1ike" feature is associated with the pulsar and is synchrotron emission we get $\mathrm{H}=8 \times 10^{-6} \mathrm{G}$ and energy in field + particles = $2 \times 10^{46}$ ergs.

The fields and energy associated with these $\mathrm{X}$-ray diffuse features are similar to those of the MSH 15-52 and Vela X synchrotron nebulae, and we can again argue that corresponding optical and radio emission will have surface brightness too low to be detectable.

In both remnants, the relatively high luminosity and the binary nature of the central system point to accretion as the most likely source of X-ray emission. The jet-like morphology of the diffuse nebulae is also quite different from that observed in the other remnants discussed.

Fig. 3 illustrates that only in these two SNR is the X-ray luminosity of the central source greater than that of the diffuse features. 
This empirical rule, at least in the X-ray band, might be useful in distinguishing accretion sources from radio pulsars. Morphology depends on orientation. W50 observed from a point on the axis of the jets would appear as a point source centered in a small diffuse nebula, and the spatial distribution of nebular emission as an indicator of type of source would be misleading.

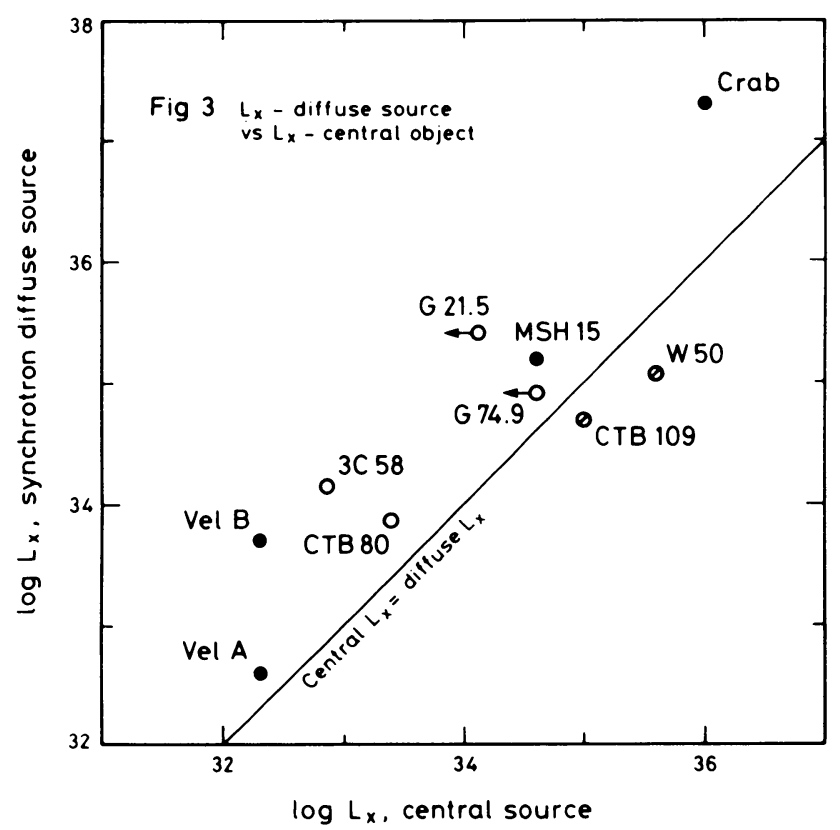

If the diffuse emission is synchrotron radiation the energy must come from a different source than electromagnetic radiation from the central pulsar. The high velocity particle beams of SS433 are an obvious choice for W50. Indeed the geometry requires this association.

Finally, it is interesting to speculate that diffuse X-ray "jets" might be a feature of most binary X-ray sources. The Radio lobes observed $\sim 2^{\prime}$ on either side of Sco X-1 (Hjelming 1971) have never been explained. Jets similar to those in w50 with $\mathrm{L}_{\mathrm{x}} \sim 6 \times 10^{34} \mathrm{erg} / \mathrm{s}$ would probably be obscured by a bright central source, and $L_{x} \sim 1038 \mathrm{erg} / \mathrm{s}$ for many binaries. Long observations of a source in eclipse or in an offstate would be capable of detecting diffuse features.

\section{SUMMARY}

The discovery of a pulsar and synchrotron nebula within MSH 15-5 2 gives a second example of a system similar to the Crab Nebula and its pulsar. Using the observed properties of MSH 15-52 we identify a likely synchrotron nebula around the Vela PSR. These three remnants show an 
Table 1 - Properties of Pulsars

\begin{tabular}{|c|c|c|c|}
\hline Pulsar & $0531+21$ & $0833-45$ & $1509-58$ \\
\hline SNR & Crab & Vela X & MSH $15-52$ \\
\hline$p(\sec )$ & $.033 \mathrm{a}$ & .089 a & $.150 \mathrm{~d}$ \\
\hline$\dot{\mathrm{p}}\left(10^{-15} \mathrm{~s} / \mathrm{s}\right)$ & $423 a$ & $125 \mathrm{a}$ & $1490 \mathrm{~d}$ \\
\hline $\mathrm{E}(\mathrm{erg} / \mathrm{s})$ & $4.6(38)$ & $6.9(36)$ & $1.7(37)$ \\
\hline m (gauss. $\left.\mathrm{cm}^{3}\right)$ & $6.7(30)$ & $6.0(30)$ & $2.6(31)$ \\
\hline age (years) & $1.23(3)$ & $1.12(4)$ & $1.66(3)$ \\
\hline $\mathrm{L}_{\mathrm{r}}\left(10^{7}-10^{8} \mathrm{~Hz}\right)(\mathrm{erg} / \mathrm{s})$ & $2(30) \mathrm{b}$ & $1(29) \mathrm{b}$ & - \\
\hline $\mathrm{L}_{\mathrm{X}}(0.2-4 \mathrm{KeV})(\mathrm{erg} / \mathrm{s})$ & $1(36) \mathrm{c}$ & $3(32) c$ & $4(34) \mathrm{e}$ \\
\hline $\mathrm{L}_{\gamma}(.05-10 \mathrm{GeV})(\mathrm{erg} / \mathrm{s})$ & $2(35) \mathrm{b}$ & $4(34) b$ & - \\
\hline $\mathrm{L}_{\text {tot }}(\mathrm{erg} / \mathrm{s})$ & $3(36)$ & $5(34)$ & $1(35)$ \\
\hline C (HRI cts/s) & 5. d & $0.25 \mathrm{c}$ & $0.01 \mathrm{e}$ \\
\hline
\end{tabular}

Table 2 - Properties of Diffuse Synchrotron Nebulae

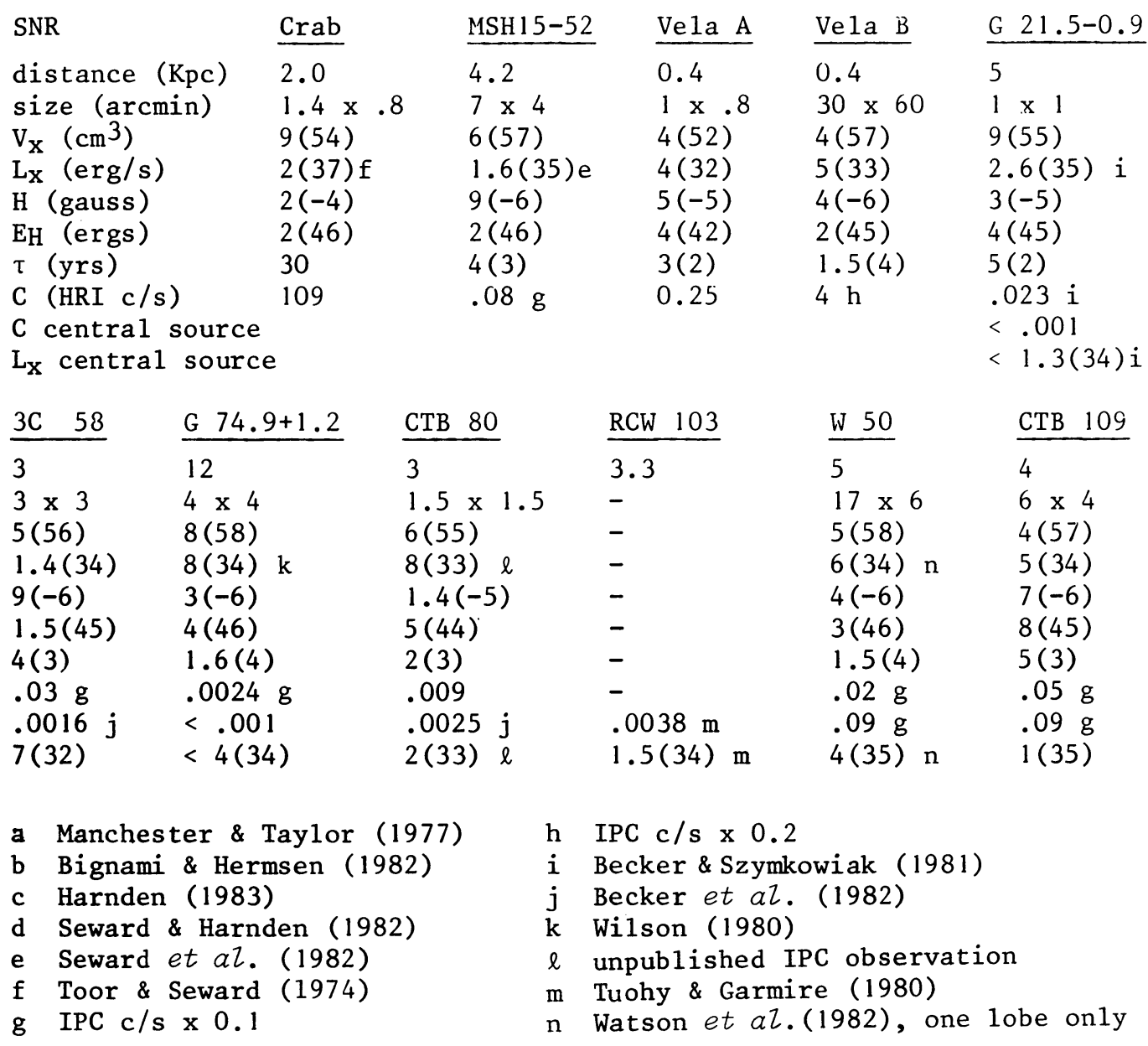


empirical connection between $\mathrm{L}_{\mathrm{X}}$. of the synchrotron nebula, $\mathrm{L}_{\mathrm{X}}$ of the central pulsar, and the pulsar $\dot{E}$. Properties of the diffuse nebula are determined by $\dot{E}$ and the size of the SNR. (The Crab is bright because $\dot{E}$ is high and because $R$ is small.) The magnetic fields derived from the observed X-ray nebulae are not far from those predicted by a simple model in which the pulsar provides the energy. The Vela pulsar is also surrounded by a smaller nebula of high surface brightness but low luminosity, analogous to the X-ray nebulae found around isolated radio pulsars. X-rays observed from 4 other filled shell radio SNR and from RCW 103 show that a central pulsar is a definite possibility. The central objects in W5O and in CTB 109 are accretion powered and more luminous than diffuse interior emission which might be synchrotron radiation. The relative strength of $X$-rays from the central source is a possible way of distinguishing true pulsars from accretion powered sources.

This work was completed while a Guest Research Fellow of the Royal Society. I would like to thank the Institute of Astronomy for their hospitality and Linda Sparke for several enlightening discussions.

\section{REFERENCES}

Becker, R. \& Szymkowiak, A. (1981) Ap.J.Letters, 248, L23.

Becker, R., Helf and, D. \& Szymkowiak, A. (1982) Ap.J., 255, 557.

Bignami, G.F. \& Hermsen, W. (1982) Ann.Rev.Ast.Ap. 21 in press.

Caswe11, J.L. (1979) MN 187, 431.

Caswe11, J.L., Milne, D. K. \& Wellington, K.J. (1981) MN 195, 89.

Chiappetti, L. \& Be11-Burne11, S.J. (1982) MN in press.

Crampton, D., Cowley, A. \& Hutchings, J. (1980) Ap.J.Letters 235, L131. Davies, R.D. \& Smith, F.G. ed. (1971) "The Crab Nebula"IAU Symposium 46. Gregory, P. (1983), this volume, p. 441.

Harnden, F.R. Jr. (1983), this volume, p. 131.

Helf and, D. (1981) IAU Symposium 95, 343.

Helf and, D. (1983), this volume, p. 483.

Hjelming, R.M. \& Wade, C.M. (1971) Ap.J.Letters 164, L1.

Lamb, R.C. \& Markert, T.H. (1981) Ap.J. 244, 94 .

Lockhart,J.A., Goss,W.M., Caswe11,J.L. \& McAdam,W.B. (1977) MN 179, 147. Manchester, R.N. \& Taylor, J.H. (1977) "Pulsars" W.H. Freeman \& Co. Manchester, R.N., Tuohy, I. \& D'Amico, N. (1982) Ap.J.Letters in press. Pravdo, S.H., Becker, R.H., Boldt, E.A., Holt, S.S., Serlemitsos, P.J. \& Swank, J.H. (1978) Ap.J. 223, 537.

Seward, F.D. \& Harnden, F.R. Jr. (1982) Ap.J.Letters 256, L45.

Seward, F.D., Harnden, F.R. Jr., Murden, P. \& Clark, D.H. (1982) submitted to Ap.J.

Smith, A. (1978) MN 182, 39P.

Toor, A. \& Seward, F.D. (1974) Ast.J. 79, 995.

Tuohy, I. \& Garmire, G. (1980) Ap.J.Letters 239, L107.

Watson, M.G., Willingale, R., Grindlay, J.E., Seward, F.D. (1982) in preparation.

Weiler, K.W. \& Shaver, P.A. (1978) Astron. \& Ap. 70, 389.

Wilson, A.S. (1980) Ap.J.Letters 241, L19. 